CP, 2018, Vol.7-No13, pp. 9/26 ISSN 2014-6752. Girona (Catalunya). Universitat de Girona. PÉREZ-RODRÍGUEZ, A.V.; GONZÁLEZ PEDRAZ, C.; ALONSO BERROCAL, J.L.: Twitter como herramienta de comunicación científica en España. Principales agentes y redes de comunicación. Recibido: 30/11/2017 - Aceptado: 13/3/2018

\title{
Twitter como herramienta de comunicación científica en España. Principales agentes y redes de comunicación
}

\section{Twitter as science communication tool in Spain. Main agents and communication networks}

\author{
Autores \\ Ana Victoria Pérez-Rodríguez \\ ORCID iD http://orcid.org/0000-0003-3608-1765 \\ Fundación Centro de Estudios para la Ciencia, la Cultura Científica y la Innovación (3CIN)/ \\ Universidad de Salamanca \\ Cristina González-Pedraz \\ ORCID iD http://orcid.org/0000-0001-8962-9224 \\ Fundación Centro de Estudios para la Ciencia, la Cultura Científica y la Innovación (3CIN)/ \\ Universidad de Valladolid \\ José Luis Alonso Berrocal \\ ORCID iD https://orcid.org/0000-0001-7470-0091 \\ Universidad de Salamanca
}

\section{Resumen}

El estudio de los principales agentes, redes de comunicación y flujos de información en Twitter es un objeto de investigación emergente. Se ha aplicado en ámbitos como la comunicación política, el deporte o el turismo, pero no a la comunicación de la ciencia. El presente trabajo está enfocado a detectar y medir a los principales agentes y redes de comunicación de la ciencia en Twitter a través de la Teoría de Redes. Se identifican los 109 usuarios personales e institucionales que están ejerciendo de influencers de la ciencia en España. Esta red se presenta como una comunidad estable y compacta. Los perfiles más productivos son los personales, lo que indica que la actividad en Twitter depende más de un interés y un compromiso individual que de disponer de un equipo de comunicación. Se detecta un uso de Twitter no tanto centrado en la difusión de contenidos y opiniones sobre ciencia, sino en la promoción de productos y eventos de divulgación. Un análisis restringido de los hashtags ha per-

\section{Abstract}

The study of the main agents, communication networks and information flows on Twitter is an emerging research topic. It has been applied in areas such as political communication, sports or tourism, but not in science communication. The present work is focused on detecting and measuring the main agents and communication networks about science on Twitter through the Network Theory. The 109 personal and institutional users who are acting as science influencers in Spain are identified. This network presents itself as a stable and compact community. The most productive profiles are the personal ones, which indicates that the activity on Twitter depends more on an interest and an individual commitment than on having a communication team. A use of Twitter is detected, not so much focused on the diffusion of contents and opinions on science, but rather on the promotion of products and events of dissemination. A restricted analysis of the hashtags has made possible to verify the strong 
mitido comprobar la fuerte vinculación de los tuits con la actualidad científica nacional e internacional. También se evidencia el especial interés que suscita Atapuerca en las conversaciones sobre ciencia en Twitter en España.

Palabras clave Comunicación científica, Twitter, redes sociales, Teoría de redes, análisis de flujos, etiquetas link between the tweets and the national and international science news. It also shows the special interest that Atapuerca arouses in the conversations about science on Twitter in Spain.

Keywords Science communication, Twitter, social networks, network theory, Flow Analysis, hashtags

\section{Introducción}

El microblogging es una forma de comunicación derivada del nuevo ecosistema de medios digitales en la que los usuarios pueden emitir mensajes instantáneos a través de sus ordenadores y dispositivos móviles. La herramienta de microblogging y red social Twitter es, sin duda, la más popular, como denota el crecimiento que ha experimentado desde su lanzamiento en octubre de 2006 (Java et al., 2007).

La característica principal de este modo de comunicación reside en la longitud de sus mensajes (Chamberlin y Lehmann, 2011; Veletsianos, 2012), reducida en el momento de la presente investigación a 140 caracteres y ampliada, en la actualidad, a 280. Por defecto, estos mensajes son públicos, aunque la red social permite difundirlos de manera privada únicamente a los seguidores de cada usuario. Twitter se utiliza para compartir información y también para describir prácticamente cualquier actividad diaria (Java et al., 2007). Además, permite a los usuarios expresar sus opiniones e intereses, de forma abreviada y altamente personalizada, en tiempo real (García-Esparza et al., 2012). Su influencia se observa en que está presente en prácticamente todas las áreas de la vida -social, política, económica, educativa, etc.- y abarca cualquier tema -deportes, cultura, ocio, industria, ciencia, etc.- (Kwak et al., 2010).

Si se valora la importancia de esta red social en términos cuantitativos, es necesario hacer referencia al volumen de tuits que se generan a diario en el mundo. Según los últimos datos ofrecidos por la propia compañía(1), los 328 millones de usuarios activos envían alrededor de 500 millones de tuits diarios. Entre ellos se encuentra un gran número de mensajes no originales que se vuelven a difundir (retuits) y que, para algunos autores, llegan a constituir nichos de opinión, ya que un mensaje creado por una persona -ya sea original o un fragmento de otra obra como un titular de un periódico o el extracto de una noticia- puede ser retuiteado por otro u otros usuarios que a su vez lo pueden volver a transmitir, provocando un efecto de expansión en forma de racimo o difusión viral (Boyd et al., 2010; Chamberlin y Lehmann, 2011).

Si bien es cierto que el contenido de gran parte de los tuis es irrelevante (Kelly, 2009) y que en muchos casos los mensajes aislados de su contexto pierden valor, Twitter es una fuente de datos muy rica ya que recoge de manera condensada información relevante para millones de usuarios, ya sean personas individuales, instituciones u otro tipo de entidades públicas y privadas, una información que resulta muy difícil de recopilar por otros canales.

Por ello, el análisis de los mensajes de Twitter está siendo utilizado como fuente primaria para múltiples investigaciones (Rogers, 2013) que abarcan desde el papel que juegan los distintos 
tipos de usuarios en la difusión de la información (Cha et al., 2012) a análisis sociológicos (Chen et al., 2012), aplicaciones a la clasificación y recuperación de la información (Lee et al., 2011; García-Esparza et al., 2012 Yerva et al., 2012) o análisis semántico (Narr et al., 2011; Alonso Berrocal et al., 2012). El estudio de sentimientos en los tuits (Thellwall, 2011; Dehkharghani et al., 2014), la credibilidad de Twitter (Castillo et al., 2011; Westerman et al., 2012) o la detección de temas relevantes a través de etiquetas o hashtags (Zhao et al., 2001; Pearce et al., 2014), son otros objetos de investigación principales en torno a esta red social.

En particular, el estudio de los agentes, sus redes de comunicación y los flujos de información en Twitter, asunto que centra la presente investigación, es una materia emergente. La aplicación de técnicas procedentes de áreas de conocimiento como las Matemáticas o la Computación en el análisis de redes sociales, como es la Teoría de Redes o de Grafos, permite identificar y medir las relaciones y flujos entre los agentes, en este caso, los distintos usuarios de Twitter, ya sean personas u entidades de todo tipo. De esta forma, es posible conocer cuáles son los usuarios de Twitter que más influyen en la comunicación en torno a determinados temas, así como las redes de comunicación que han tejido.

Diversos trabajos han tratado de medir la influencia de los agentes en Twitter (Berry, 2010; Edwards, 2013) así como los flujos y redes de comunicación en torno a eventos determinados, tales como los Juegos Olímpicos de Londres de 2012 (Willis et al., 2015) o la defensa del Parque Gezi en Turquía (Ogan y Varol, 2017), o temas diversos como las comunidades políticas canadienses (Dubois y Gaffney, 2014) o austriacas (Ausserhofer y Maireder, 2013), la gestión de crisis humanitarias (Lozano y Vaca, 2017) o las valoraciones y opiniones en Twitter sobre el sector turístico (Barbagallo et al., 2012).

\subsection{Twitter como herramienta de comunicación científica}

En el ámbito específico de la comunicación de la ciencia, Twitter está teniendo una influencia significativa. Según la última Encuesta de Percepción Social de la Ciencia de FECYT, correspondiente a 2016(2), Internet fue el medio de comunicación para informarse sobre ciencia y tecnología más citado en primer lugar por la población española. Dentro de Internet, el 75,4\% de los encuestados manifestó informarse a través de redes sociales, un porcentaje que ha crecido exponencialmente desde 2010, cuando solo el 18,4\% afirmó informarse sobre ciencia y tecnología por esta vía. En concreto, el 37,3\% lo hace a través de Twitter, un porcentaje que se eleva hasta el $51,9 \%$ en la franja de edad de 15 a 24 años.

En la literatura, diversos trabajos han abordado las redes sociales en general, y Twitter, en particular, como herramienta para la comunicación de la ciencia. Como apunta Ribas (2012), las personas que se dedican a la comunicación de la Ciencia utilizan cada vez más la tecnología digital y las redes sociales. Muchos periodistas de ciencia promocionan los artículos que escriben a través de las redes sociales, las universidades y centros de investigación distribuyen a través de ellas sus comunicados de prensa, y los científicos destacan sus publicaciones con el objetivo de ganar en impacto (Peters et al., 2014). A ellos se suman otros actores, expertos y no expertos, que participan en este sistema cooperativo (De Semir, 2010) y favorecen la multiplicación y el efecto viral de los mensajes de temática científica y tecnológica (Fernández de Lis, 2013). Hoy en día, los primeros datos sobre cualquier primicia científica o tecnológica se hacen públicos a través de Twitter (Brown Jarreau, 2014). Incluso, los propios profesionales del periodismo 
de ciencia utilizan las redes sociales para estar informados de las últimas novedades, para mantener un contacto directo con las fuentes y para contrastar informaciones en tiempo real (Pont Sorribes et al., 2013). Según los mismos autores, Twitter (96\%) es la red social más utilizada por los periodistas de ciencia y su semejanza con la recepción de teletipos es una de las razones que más contribuyen a su uso.

Así, gran parte de los trabajos que analizan el uso de redes sociales en la comunicación de la ciencia se centran en Twitter. Precisamente, cómo los periodistas y los gabinetes de comunicación de los centros de investigación utilizan esta y otras redes sociales (Montenegro y Escudero, 2013; Kahle et al., 2016; Quiñónez Gómez y Sánchez Colmenares, 2016) centra diversos estudios. También el uso que científicos y académicos hacen de Twitter (Bonetta, 2009; Nentwich y König, 2014) o el impacto de esta red social en relación con la publicación científica (Mandavili, 2011; Liang et al. 2014).

El discurso público en Twitter y la percepción del riesgo sobre temas que suscitan algún tipo de controversia, tales como la nanotecnología o la energía nuclear, son objeto de otros trabajos (Veltri, 2012; Runge et al., 2013; Li et al., 2016).

En cambio, no se ha localizado ningún estudio enfocado a tratar de detectar y medir los principales agentes y redes de comunicación de la ciencia en Twitter, aplicando la Teoría de Grafos, como sí se ha intentado en otros ámbitos.

Por ello, el objetivo principal del presente estudio es analizar Twitter como herramienta de comunicación de la ciencia, acotando el trabajo al caso español, para localizar los agentes más importantes o influencers y conocer sus flujos de información, a partir de dicha Teoría. Aunque en las redes sociales online se ha generalizado en los últimos tiempos la utilización del término influencer para referirse a estos agentes influyentes, este perfil se asimila a los tradicionales líderes de opinión (Lazarsfeld et al., 1944) estudiados ampliamente en otros ámbitos como la sociología (Gitlin, 1978; Burt, 1999; Del Fresno et al., 2016) o el marketing (King y Summers, 1970; Kiss y Bichler, 2008; Brown y Hayes, 2008).

Como objetivos subordinados se plantean los siguientes: medir la cantidad e intensidad de las relaciones que se establecen entre los 100 agentes más productivos de la red, determinar qué tipos de perfiles (personales o institucionales) son los más activos y cuáles son los más retuiteados (los que alcanzan una mayor visibilidad), establecer cuáles son los temas o topics científicos que centran la atención de los usuarios de Twitter en España y estimar el Índice $\mathrm{h}$ de popularidad en Twitter de estos agentes.

Se busca así responder a las siguientes preguntas de investigación:

P1. ¿Cuáles son los perfiles más influyentes en la comunicación de la ciencia en España a través de Twitter?

P2. ¿Cuáles son las principales redes de comunicación que han tejido estos agentes?

P3. ¿Qué tipos de perfiles son los más activos y cuáles obtienen una mayor visibilidad?

P4. ¿Cuáles son los temas científicos más comentados a través de Twitter en España?

P5. ¿Cuáles son los perfiles con mayor Índice h de popularidad en Twitter en el país? 


\section{Metodología}

La aplicación de la Teoría de Grafos o de Redes a este caso consiste en generar, a partir de una serie de datos brutos descargados de Twitter y procesados a tal efecto, una estructura gráfica formada por nodos o vértices, que representan a los agentes (perfiles), y aristas, que simbolizan las relaciones entre los nodos (Dehmer et al., 12015; Akhtar et al., 2017). Los conceptos de centralidad e intermediación juegan un papel clave en la metodología planteada. La intermediación es una medida de centralidad que ofrece una idea del prestigio o poder que tienen los actores que intervienen en la red analizada. Así, los nodos con un valor elevado tienen mucho poder, pues controlan los flujos de información.

Para iniciar el estudio se optó por componer una muestra inicial de 50 agentes, de cuyo análisis masivo se obtendrían los 100 perfiles más significativos que compondrían la muestra final. La muestra inicial fue propuesta por un comité de expertos conformado por seis académicos y divulgadores científicos(3), quienes consensuaron los 50 perfiles personales e institucionales especializados en la difusión de contenidos científicos que serían objeto del estudio preliminar.

Para ello, el comité de expertos se reunió en tres sesiones celebradas entre los meses de abril a junio de 2014. Cada uno de los miembros del panel propuso los perfiles considerados más representativos, atendiendo a tres criterios principales: adecuación (que efectivamente se tratase de perfiles dedicados fundamentalmente a la divulgación de la ciencia); implantación (considerando solamente aquellos perfiles con un número mínimo de 3.000 seguidores) y representatividad (la muestra debía incluir perfiles de varios tipos institucionales relevantes para el estudio (medios de comunicación, agencias de noticias, universidades centros de investigación, fundaciones) así como perfiles personales (periodistas, divulgadores, investigadores, etc.). De los perfiles propuestos por los expertos se incluyeron directamente aquellos en los que existía consenso o que fueron seleccionados por al menos cuatro de los seis integrantes del grupo de trabajo. Los perfiles restantes hasta acordar los 50 que finalmente integraron la muestra inicial fueron consensuados durante las sesiones de trabajo.

A través de la interfaz de programación de búsqueda de Twitter (Twitter Search API), se descargó la actividad de estos 50 perfiles entre el 1 de enero y el 30 de septiembre de 2015 y se cuantificaron las menciones presentes en sus tuits. Finalmente, se identificaron los 109 perfiles mencionados con mayor frecuencia y en menor espacio de tiempo, considerados los más significativos.

Posteriormente, se descargó durante los mismos nueve meses la actividad de estos 109 agentes principales que conformaron la muestra final. Esta descarga incluyó todos los tuits emitidos por cada perfil en las fechas de la consulta. Parte del análisis se ha realizado directamente a partir de los datos brutos mediante programas específicos. Paralelamente, se obtuvo un fichero de texto plano delimitado según el formato JSON (JavaScript Object Notation), un formato ligero de intercambio de datos que es uno de los estándares más utilizados y cuya sintaxis permite identificar objetos y parejas clave:dato (Lanthaler y Gütl, 2013; Sriparasa, 2013). Los objetos están bien documentados por Twitter, cuyo diccionario de datos especifica la lista de claves de cada objeto y su uso. 
Conceptualmente, estos objetos y parejas se corresponden con los campos y valores de las tablas de una base de datos, pero formalmente son más bien datos brutos que requieren algún tipo de procesado para poder abordar su análisis.

De este modo, para el presente estudio se ha desarrollado un programa que convierte el formato JSON en tablas de una base de datos relacional SQL, en la que sus tablas, campos y datos representan a los objetos, claves y valores del formato JSON, y las relaciones entre las tablas reflejan la estructura jerárquica entre los objetos.

A partir de los datos recuperados se han analizado los siguientes parámetros:

- Cantidad de tuits de contenido científico generados.

- Evolución temporal.

- Tipos de perfiles más activos y tipos de perfiles más retuiteados.

- Alcance de los contenidos a través de un análisis de flujo.

- Asuntos científicos de mayor interés en función de los topics identificados.

\subsection{Obtención del Î́ndice $\mathrm{h}$}

Desde el surgimiento de Twitter en 2006 se han definido diversas metodologías, bien con orientación académica, bien con orientación comercial o publicitaria, para medir la relevancia de los perfiles (agentes). Las más sencillas se basan en el recuento de seguidores, pero su prestigio y fiabilidad ha ido decreciendo a medida que se ha profesionalizado y extendido el uso de esta red social, debido a que es práctica habitual entre los usuarios que desean explotar la notoriedad de su perfil definir estrategias para incrementar sus seguidores.

Con el fin de obtener una visión más real de la influencia que ejercen determinados perfiles en un clúster determinado, los expertos en métricas aplican desde hace unos años una metodología similar a la utilizada en el ámbito académico para establecer la relevancia de autores y publicaciones científicas. Por este motivo se la denomina, en clara referencia a la fórmula bibliométrica en la que se inspira, Índice h de popularidad en Twitter (Razis y Anagnostopoulos, 2016).

El resultado de la aplicación de dicha fórmula en el presente objeto de estudio es un número que se asocia a cada uno de los perfiles analizados y que se interpreta de la siguiente manera: si una cuenta posee un Índice h de 7, significa que ha emitido al menos 7 tuits, cada uno de los cuales ha sido retuiteado por lo menos en 7 ocasiones. De este modo, la fórmula permite obtener una imagen de cuáles son los perfiles cuya producción consigue un impacto medio mayor.

\section{Resultados}

\subsection{Datos generales}

Desde el 1 de enero hasta el 30 de septiembre de 2015 los perfiles de la muestra emitieron un total de 342.296 mensajes, de los cuales 234.854 se corresponden con tuits originales y 107.189 son retuits de mensajes procedentes de otros perfiles. Ello supone un índice de actividad media para el conjunto de la muestra próximo a los 40.000 tuits mensuales.

La evolución de la actividad registrada mes a mes revela que ésta se mantiene estable a lo largo del periodo estudiado, contabilizándose un descenso pronunciado cercano a los 11.000 tuits durante el mes de agosto, coincidiendo con las vacaciones estivales. 
El análisis de los retuits ha resultado clave para la identificación de aquellos perfiles que tanto en términos de actividad total (tuits+retuits) como de producción propia (tuits) se han mostrado relevantes en el ámbito de la comunicación de la ciencia en España. A partir del análisis de dichos mensajes se ha identificado un total de 25.000 perfiles que han retuiteado, al menos una vez, alguno de los mensajes emitidos por los perfiles de la muestra.

\section{Gráfico 1. Evolución mensual de las cifras de producción y actividad total}

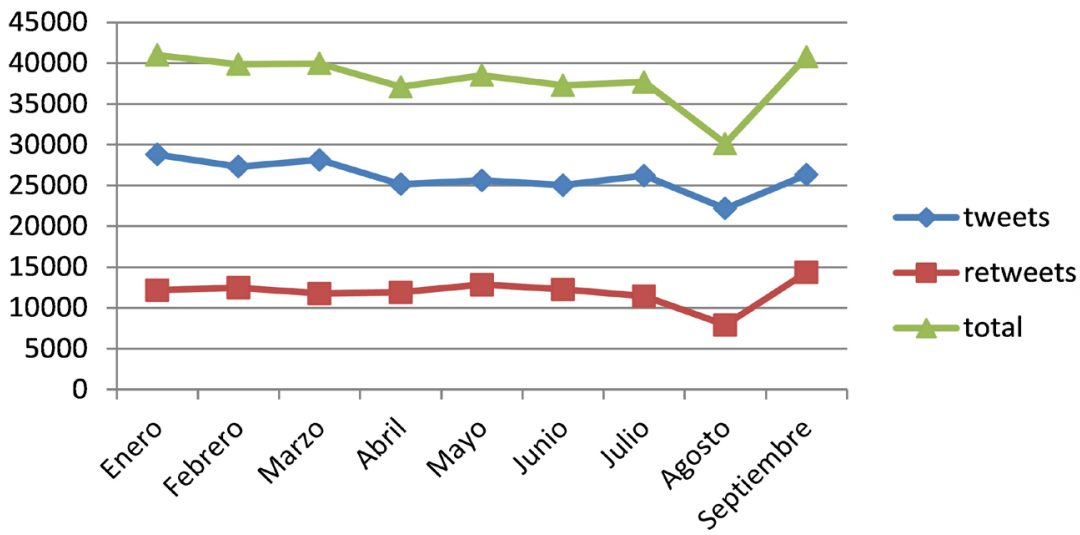

Fuente: elaboración propia.

Mucho mayor es el número de perfiles mencionados en los tuits ya que, descontando las automenciones, se han contabilizado de enero a septiembre más de 82.800 referencias a otros usuarios.

\subsection{Tipos de perfiles más activos y tipos de perfiles más retuiteados}

Para cuantificar los porcentajes de actividad de los distintos tipos de agentes implicados en la comunicación de la ciencia a través de Twitter, se definieron un total de 13 categorías, en función de las cuales se codificaron posteriormente los 109 perfiles de la muestra final: asociaciones científicas, centros de investigación, fundaciones, empresas, medios de comunicación, museos, universidades, programas de TV, científicos, divulgadores, blogueros, periodistas o usuarios individuales (tuiteros).

Los científicos, con un 37\% de la actividad total, son el tipo de agente más destacado en la comunicación de la ciencia en Twitter, seguido, muy por detrás, por periodistas (19\%) y medios de comunicación (15\%). Estos tres tipos de agentes han emitido el $71 \%$ del total de tuits y retuits objeto de análisis. El 29\% restante se lo reparten, en pequeños porcentajes, los otros 10 tipos de agentes especificados. 


\section{Gráfico 2. Porcentaje de actividad total por tipos de perfil}

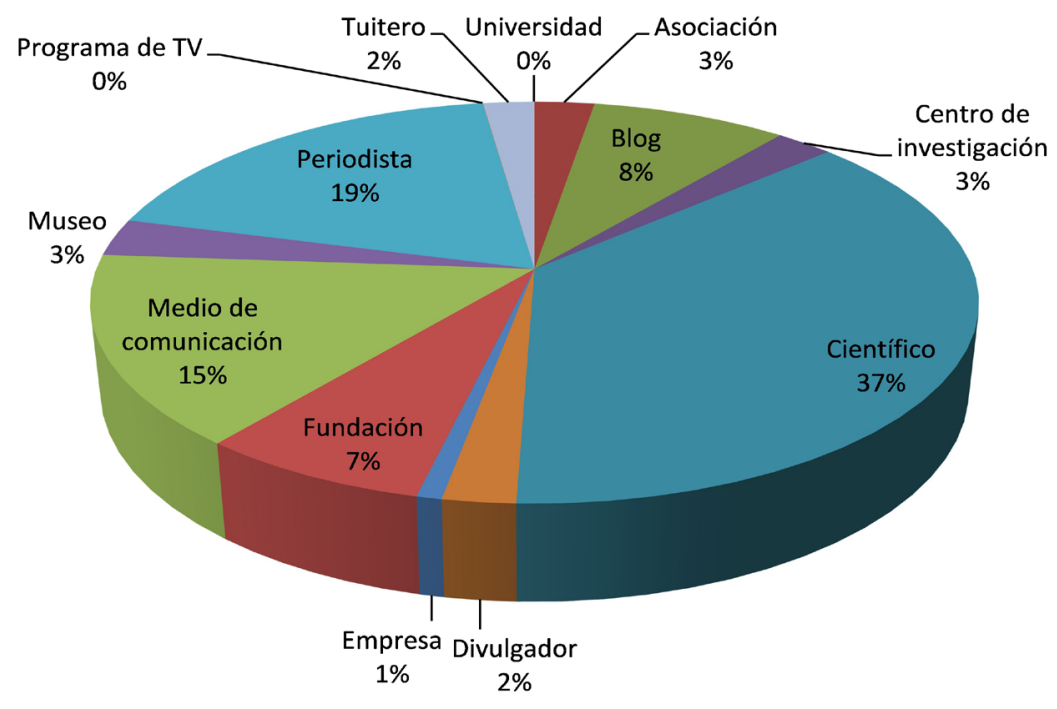

Fuente: elaboración propia.

Resulta significativo cómo los perfiles personales (científicos y periodistas) son más activos que los perfiles institucionales, teniendo en cuenta que entidades como universidades, centros de investigación o museos de ciencia cuentan con gabinetes de comunicación y difusión que se encargan, entre otras tareas, de la comunicación digital corporativa (Gerber, 2014).

Por otro lado, se ha estimado qué tipos de perfiles son los más retuiteados, es decir, qué tipos de perfiles son los que alcanzan una mayor visibilidad. Aunque de nuevo científicos (18\%) y periodistas (14\%) son los que alcanzan unas cifras mayores, los porcentajes están más equilibrados con el resto de agentes. Blogueros (13\%), medios de comunicación (12\%) y divulgadores (10\%) se encuentran también entre los más visibles. Asimismo, se ha observado que diversos eventos de carácter científico o tecnológico son objeto del $10 \%$ de retuits recogidos.

La diferencia entre los porcentajes de actividad total y de retuits pone de manifiesto que algunos tipos de agentes son muy eficaces en la comunicación de la ciencia a través de Twitter: emiten pocos mensajes pero son muy retuiteados, lo que les permite obtener una gran difusión y visibilidad. Es el caso de los divulgadores ( $2 \%$ de la actividad total y $10 \%$ de retuits), las universidades (menos del $1 \%$ de la actividad total y $6 \%$ de retuits) y de los blogueros ( $8 \%$ de la actividad total y $13 \%$ de retuits).

\subsection{Análisis de flujo}

El análisis de flujo ha permitido medir la cantidad y la intensidad de las relaciones establecidas entre los 109 agentes más productivos de la red. Tal y como muestra la representación gene- 
rada, se trata de un clúster muy centralizado con una serie de nodos nucleares fuertemente conectados entre sí a los que se asocian, por distintas vías, varias comunidades periféricas.

\section{Gráfico 3. Red de agentes (nodos) y relaciones establecidas (aristas)}

Fuente: elaboración propia.

La imagen permite identificar aquellos nodos clave que articulan el sistema. En este caso, destacados en color, se identifican los perfiles de @fecyt_ciencia (rojo); @jmmulet (naranja); @a_valenzuela (amarillo) y @aberron (verde). Estos cuatro perfiles, en función de su grado de intermediación, concentran buena parte del tráfico informativo observado en la red, lo que los convierte en agentes clave o influencers a la hora de garantizar una mayor visibilidad de los mensajes emitidos por el resto de nodos.

Por otra parte, la aproximación a cada una de las comunidades satélite ofrece información acerca de los nodos clave que articulan la circulación de contenidos en cada una de ellas. Por ejemplo, en la parte inferior de la imagen, se observa una importante comunidad satélite con un nodo principal (@fatapuerca, en amarillo) y tres nodos secundarios colindantes (@mu- 
seoevolucion, @miguelonmeh y @juanluisarsuaga) fuertemente conectados entre sí y con los nodos centrales. De este modo, es posible establecer que son estos cuatro perfiles los agentes clave en la circulación de información relacionada con la investigación y la difusión sobre evolución humana y, en concreto, sobre los yacimientos de Atapuerca.

\subsection{Hashtags}

Los hashtags o etiquetas utilizadas por los usuarios de Twitter para identificar el tema acerca del que gira un mensaje y facilitar su difusión ha sido otro de los objetos de análisis. Su uso generalizado por parte de los usuarios que operan en esta red social los convierte en una herramienta interesante para detectar aquellos temas o topics que centran la atención de una comunidad (Antenucci et al., 2011).

Con este propósito se identificaron un total de 19.543 hashtags en el conjunto de la muestra. La información recuperada para cada una de esas etiquetas incluye la fecha de emisión, así como el número de menciones recibidas para esa fecha. De este modo, ha sido posible medir y ordenar los hashtags más populares para cada una de las 36 semanas que integran el periodo de estudio. Debido al gran volumen total de hashtags, se consideraron únicamente aquellos que habían recibido al menos 11 menciones.

\section{Gráfico 4. Hashtags de temática científica más mencionados.}

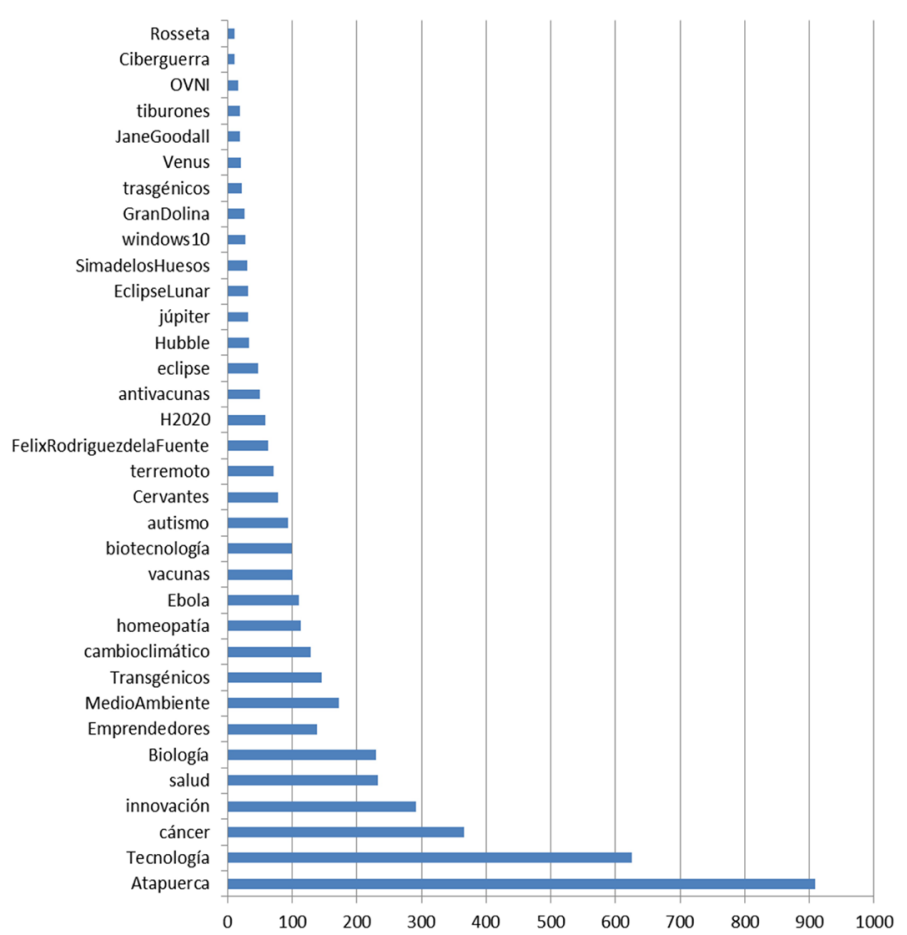

Fuente: elaboración propia. 
La mayor parte de los hashtags más mencionados tiene un carácter netamente promocional. O bien hace referencia a productos comunicativos (programas de TV, blogs, libros, etc.), o bien a eventos de divulgación (La Noche de los Investigadores, Museum Week, etc.). El predominio de este tipo de etiquetas resulta coherente con el análisis de los retuits expuesto anteriormente, en el que se advertía el peso de los eventos de divulgación científica dentro del volumen total de retuits (10\%).

Una consecuencia derivada del predominio del uso de Twitter como herramienta de promoción es que la identificación de los hashtags no ha sido, en el caso concreto del clúster identificado, una metodología útil para la detección de aquellos temas científicos sobre los que más diserta dicha comunidad. Por ello, se procedió a una revisión manual de hashtags, lo que hizo posible identificar un total de 34 etiquetas referentes a temáticas científicas y tecnológicas.

Atapuerca destaca notablemente como el tema científico que suscita un mayor interés en la comunidad estudiada. El hashtag \#Atapuerca, con más de 900 menciones, es el asunto de carácter científico más referenciado en el periodo de análisis. En el ránking de etiquetas más mencionadas se encuentran también \#SimadelosHuesos y \#GranDolina, dos de los yacimientos de la Sierra. Asimismo, temas que originan controversia como los transgénicos, el cambio climático, la homeopatía o la asociación entre vacunas y autismo, también han centrado el debate en Twitter en el periodo estudiado.

Por otro lado, la revisión de las fechas en las que estos hashtags alcanzan su máximo de menciones indica una clara vinculación de las etiquetas con la actualidad informativa del periodo de análisis. Es el caso de etiquetas como \#ebola, \#antivacuna, \#Cervantes o \#JaneGoodall. Las menciones del hashtag \#ebola se concentran fundamentalmente en el mes de enero de 2015, momento en que los medios de comunicación aún daban una amplia cobertura al caso de la enfermera española contagiada en el Hospital Carlos III de Madrid. La paciente fue oficialmente dada de alta en noviembre de 2014, aunque la polémica acerca de cómo pudo contagiarse, las consecuencias de dicho contagio y la gestión administrativa y sanitaria del caso se mantuvieron vigentes hasta bien entrado el mes de enero.

Asimismo, el hashtag \#antivacuna es uno de los más mencionados en el mes de junio de 2015, momento en que se produjo la noticia del fallecimiento de un niño español afectado de difteria que no había sido vacunado por decisión de sus padres. También los hashtag \#Cervantes, en referencia a las investigaciones realizadas para determinar si los restos encontrados en una cripta madrileña eran los del escritor o \#JaneGoodall, predominante durante la visita de la primatóloga a España, muestran una clara vinculación con la actualidad del momento.

\section{5. Índice h de popularidad en Twitter}

La fórmula aplicada para medir el Índice h de popularidad en Twitter ha permitido obtener un listado de los perfiles cuya producción propia consigue un impacto medio mayor.

Un total de 12 perfiles obtiene un Índice h mayor de 60. Los dos primeros se corresponden con usuarios que se dedican, de distinta forma, a la divulgación científica en la televisión. Tres medios de comunicación, Muy Interesante, Materia y ABC; y dos blogs, Tapas de Ciencia y Microsiervos, se encuentran también entre los perfiles más populares. Solo un organismo de 
investigación, el CSIC, aparece en los primeros lugares.

Gráfico 5. Ránking de perfiles con mayor Índice h

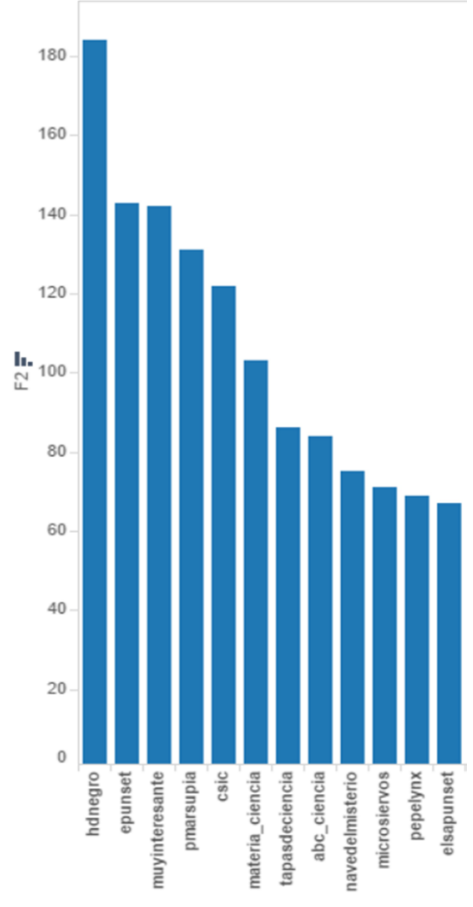

Fuente: elaboración propia.

\section{Conclusiones y discusión}

El presente trabajo ha aplicado, por primera vez, la Teoría de Redes o de Grafos para determinar los principales agentes y redes de comunicación de la ciencia en Twitter en España.

En cuanto a los agentes, la detección de los usuarios más influyentes es un tema clave ya que desempeñan un papel fundamental en la difusión de la información (Simmie et al., 2014). En este caso, se ha podido conocer qué agentes principales están articulando la comunicación de la ciencia a través de Twitter y ejercen como influencers respecto a la difusión de contenidos y opiniones de carácter científico y tecnológico en el país.

Asimismo, el análisis de los flujos de información que se establece entre los diferentes agentes ha ofrecido datos interesantes acerca de las dinámicas que rigen la actividad del clúster en su conjunto, y también acerca de la función que desempeña cada uno de sus nodos.

La red de perfiles principales ligados a la comunicación de la ciencia en España se presenta como una comunidad estable, tanto en número de usuarios como en términos de actividad, lo que puede constituir una de las claves de su éxito. La estabilidad observada tanto en las cifras 
de producción, como en las de actividad y en las de visibilidad de los distintos tipos de perfiles definidos son indicios que apuntan a que el clúster de agentes vinculados al ámbito de la comunicación de la ciencia en España es sólido y compacto.

El hecho de que los perfiles más productivos sean los personales y no institucionales apunta hacia una nueva hipótesis, y es que disponer de un equipo de márketing o comunicación institucional no parece ser sinónimo de mayor actividad en Twitter, sino que esta depende más de un interés y un compromiso personal. No obstante, serán necesarios más estudios específicos sobre esta materia. Asimismo, las diferencias entre los porcentajes de la actividad total y de retuits que obtienen algunos tipos de perfiles sugiere que algunos agentes son enormemente eficaces en su difusión.

Por otro lado, el ranking de hashtags más mencionados, el volumen de retuits y los perfiles con mayor Índice h apuntan a un uso de Twitter no tanto centrado en la difusión de contenidos y opiniones de corte científico, sino en la promoción de productos y eventos de divulgación. Esta tendencia se muestra también en el análisis de flujo, que ha permitido identificar como prescriptores relevantes no a los científicos más prestigiosos o a las instituciones de investigación de mayor tamaño en España, sino a conocidos comunicadores y divulgadores y a las entidades más activas en la promoción de la Cultura Científica en el país, como son FECYT y sus estructuras afines, la Agencia SINC o el Museo Nacional de Ciencia y Tecnología.

Un análisis restringido de los hashtags ha permitido comprobar la fuerte vinculación de los temas tratados con la actualidad científica nacional e internacional. El estudio de las etiquetas y el análisis de flujo han mostrado además la pujanza de la investigación sobre evolución humana y los yacimientos de Atapuerca en las conversaciones sobre ciencia en Twitter en España.

Próximos estudios deberán enfocarse hacia muestras más amplias y a estudiar nodos y asuntos científicos particulares, así como a contrastar si los resultados obtenidos en España son extrapolables a las realidades presentes en otros países y contextos.

\section{Financiación}

El artículo presentado se enmarca en los trabajos desarrollados en el proyecto 'Cultura Científica 2.0: Análisis de contenidos de cultura científica en Twitter', financiado por la Fundación Española para la Ciencia y la Tecnología (FECYT) en 2015 [Ref. FCT-14-9255]. Página web del proyecto: http://berrocal.usal.es/fecyt/intermediacion.html

\section{Agradecimientos}

Los autores del presente trabajo agradecen la colaboración de los seis académicos y divulgadores científicos que participaron de forma altruista y anónima en el comité de expertos que seleccionó los 50 perfiles iniciales con los que pudo obtenerse la muestra final objeto de estudio.

\section{Referencias bibliográficas}

- $\quad$ Akhtar, N., \& Ahamad, M. V. (2017). Graph Tools for Social Network Analysis. Graph Theoretic Approaches for Analyzing Large-Scale Social Networks, 18.

- Alonso Berrocal, J. L., Gómez Díaz, R., Figuerola, C. G., Zazo Rodríguez, Á. F., y Cordón 
García, J. A. (2012). "Propuesta de estudio del campo semántico de los libros electrónicos en Twitter". Scire, 18(2), 87-97.

- Antenucci, D., Handy, G., Modi, A., y Tinkerhess, M. (2011). "Classification of tweets via clustering of hashtags". EECS, 545, 1-11.

- $\quad$ Ausserhofer, J., y Maireder, A. (2013). "National politics on Twitter: Structures and topics of a networked public sphere". Information, Communication \& Society, 16(3), 291-314.

- Barbagallo, D., Bruni, L., Francalanci, C., y Giacomazzi, P. (2012). "An Empirical Study on the Relationship between Twitter Sentiment and Influence in the Tourism Domain". En: Information and Communication Technologies in Tourism 2012 (pp. 506-516). Springer, Vienna. Boyd, D., Golder, S., y Lotan, G. (2010). "Tweet, tweet, retweet: Conversational aspects of retweeting on twitter". En: System Sciences (HICSS), 2010 43rd Hawaii International Conference.

- $\quad$ Bonetta, L. (2009). "Should you be tweeting?". Cell, 139(3), 452-453

- $\quad$ Brown Jarreau, P. (2014). An explosion of alternatives. EMBO reports, e201439130.

- Brown, D., y Hayes, N. (2008). Influencer marketing. Who really influences your customers? Routledge.

- $\quad$ Burt, R. S. (1999). "The social capital of opinion leaders". The Annals of the American Academy of Political and Social Science, 566(1), 37-54.

- Castillo, C., Mendoza, M., y Poblete, B. (2011). "Information credibility on twitter". En: Proceedings of the 20th international conference on World wide web (pp. 675-684). ACM.

- Cha, M., Benevenuto, F., Haddadi, H., y Gummadi, K. (2012). "The world of connections and information flow in twitter". IEEE Transactions on Systems, Man, and Cybernetics-Part A: Systems and Humans, 42(4), 991-998.

- Chamberlin, L., y Lehmann, K. (2011). "Twitter in higher education". Cutting-edge Technologies in Higher Education, 1, 375-391.

- $\quad$ Chen, S.-C., Yen, D. C., y Hwang, M. I. (2012). "Factors influencing the continuance intention to the usage of Web 2.0: An empirical study". Computers in Human Behavior, 28(3), 933-941.

- De Semir, V. (2010). "El «mutatis mutandis» de la comunicación científica en la era de Internet". ArtefaCToS, 3(1), 49-79. Disponible en:

- Dehkharghani, R., Mercan, H., Javeed, A., \& Saygin, Y. (2014). Sentimental causal rule discovery from Twitter. Expert Systems with Applications, 41(10), 4950-4958.

- Dehmer, M., Emmert-Streib, F., y Pickl, S. (2015). Computational Network Theory: Theoretical Foundations and Applications (Vol. 5). John Wiley \& Sons.

- Del Fresno Garcia, M., Daly, A. J., y Segado Sanchez-Cabezudo, S. (2016). "Identifying the new Influences in the Internet Era: Social Media and Social Network Analysis". Revista Española de Investigaciones Sociológicas, 153: 23-40.

- Dubois, E., y Gaffney, D. (2014). "The multiple facets of influence: identifying political influentials and opinion leaders on Twitter." American Behavioral Scientist, 58(10), 1260-1277.

- Fernández de Lis, P. (2013). "Ciencia y periodismo en la red". Quaderns de la Fundació Dr. Antoni Esteve, 28, 15-19.

- $\quad$ Garcia Esparza, S., O’Mahony, M. P., y Smyth, B. (2012). "Mining the real-time web: a novel approach to product recommendation". Knowledge-Based Systems, 29, 3-11.

- Gerber, A. (2014). "Science Caught Flat-Footed: How Academia Struggles with Open Science Communication". En: Bartling, S., y Friesike, S. Opening Science. Springer International Publishing. Págs 73-80. 
- Java, A., Song, X., Finin, T., y Tseng, B. (2007). "Why we twitter: understanding microblogging usage and communities". En: Proceedings of the 9th WebKDD and 1st SNA-KDD 2007 workshop on Web mining and social network analysis (pp. 56-65). ACM.

- Kahle, K., Sharon, A. J., y Baram-Tsabari, A. (2016). "Footprints of Fascination: Digital Traces of Public Engagement with Particle Physics on CERN's Social Media Platforms". PloS one, 11(5), e0156409

- Kelly, R. (2009). "Twitter study reveals interesting results about usage". PearAnalytics. August 12th.

- King, C. W., y Summers, J. O. (1970). "Overlap of opinion leadership across consumer product categories". Journal of Marketing Research, 43-50.

- Kiss, C., y Bichler, M. (2008). "Identification of influencers-measuring influence in customer networks". Decision Support Systems, 46(1), 233-253.

- Kwak, H., Lee, C., Park, H., y Moon, S. (2010). "What is Twitter, a social network or a news media?". En: Proceedings of the 19th international conference on World wide web (pp. 591600). ACM.

- Lanthaler, M., y Gütl, C. (2012). "On using JSON-LD to create evolvable RESTful services". En: Proceedings of the Third International Workshop on RESTful Design (pp. 25-32). ACM.

- Lee, K., Palsetia, D., Narayanan, R., Patwary, M. M. A., Agrawal, A., y Choudhary, A. (2011). "Twitter trending topic classification". Paper presented at the Data Mining Workshops (ICDMW), IEEE 11th International Conference.

- $\quad$ Liang, X., Su, L. Y. F., Yeo, S. K., Scheufele, D. A., Brossard, D., Xenos, M., ... y Corley, E. A. (2014). "Building Buzz (Scientists) Communicating Science in New Media Environments". Journalism \& Mass Communication Quarterly.

- $\quad$ Li, N., Akin, H., Su, L. Y. F., Brossard, D., Xenos, M., y Scheufele, D. A. (2016). "Tweeting disaster: an analysis of online discourse about nuclear power in the wake of the Fukushima Daiichi nuclear accident. JCOM, 15(05), A02-2.

- Lozano, E., y Vaca, C. (2017). "Crisis management on Twitter: Detecting emerging leaders". En: eDemocracy \& eGovernment (ICEDEG), 2017 Fourth International Conference on (pp. 140-147). IEEE.

- Mandavilli, A. (2011). "Trial by twitter". Nature, 469 (7330), 20.

- Montenegro, V., y Escudero, H. (2013). "Las redes sociales y la difusión de la tecnología y la innovación". En: III Congreso Internacional de Comunicación Pública de la Ciencia, Santa $\mathrm{Fe}$, Argentina.

- $\quad$ Narr, S., De Luca, E. W., y Albayrak, S. (2011). "Extracting semantic annotations from twitter". En: Proceedings of the fourth workshop on Exploiting semantic annotations in information retrieval.

- Nentwich, M., y König, R. (2014). "Academia Goes Facebook? The Potential of Social Network Sites in the Scholarly Realm". En: Bartling, S., y Friesike, S. Opening Science. 107-124. Springer International Publishing.

- Ogan, C., y Varol, O. (2017). "What is gained and what is left to be done when content analysis is added to network analysis in the study of a social movement: Twitter use during Gezi Park". Information, Communication \& Society, 20(8), 1220-1238.

- Pearce, W., Holmberg, K., Hellsten, I., y Nerlich, B. (2014). "Climate change on Twitter: Topics, communities and conversations about the 2013 IPCC Working Group 1 report". PloS one, 9(4), e94785.

- $\quad$ Peters, H. P., Dunwoody, S., Allgaier, J., Lo, Y. Y., y Brossard, D. (2014). "Public communica- 
tion of science 2.0". EMBO reports, 15(7), 749-753.

- $\quad$ Pont Sorribes, C., Cortiñas Rovira, S., y Di Bonito, I. (2013). "Challenges and opportunities for science journalists in adopting new technologies: the case of Spain". JCOM, 12 (03).

- Quiñónez Gómez, H., y Sánchez Colmenares, M. (2016). “Uso de Twitter en el periodismo científico. Los casos de los diarios El Nacional y El Universal en Venezuela". Questión, 1.

- Razis, G., y Anagnostopoulos, I. "InfluenceTracker: Rating the Impact of a Twitter Account". (2016). En: Iliadis, L., Maglogiannis, I., Papadopoulos, H., Sioutas, S., y Makris, C. Artificial Intelligence Applications and Innovations. Springer International $\mathrm{Pu}$.

- Ribas, C. (2012). "La divulgación y la comunicación de la ciencia, en la encrucijada". SEBBM, 173, 10-12.

- Rogers, R. (2013, May). "Debanalizing Twitter: The transformation of an object of study". En: Proceedings of the 5th Annual ACM Web Science Conference, 356-365. ACM.

- $\quad$ Runge, K. K., Yeo, S. K., Cacciatore, M., Scheufele, D. A., Brossard, D., Xenos, M., y Su, L. Y. F. (2013). "Tweeting nano: How public discourses about nanotechnology develop in social media environments". Journal of nanoparticle research, 15(1), 1-11.

- Simmie, D., Vigliotti, M. G., y Hankin, C. (2014). "Ranking twitter influence by combining network centrality and influence observables in an evolutionary model". Journal of Complex Networks, 2(4), 495-517.

- $\quad$ Sriparasa, S. S. (2013). JavaScript and JSON essentials. Packt Publishing Ltd.

- Veletsianos, G. (2012). "Higher education scholars' participation and practices on Twitter". Journal of Computer Assisted Learning, 28(4), 336-349.

- Veltri, G. A. (2013). Microblogging and nanotweets: Nanotechnology on Twitter. Public Understanding of Science, 22(7), 832-849.

- Westerman, D., Spence, P. R., y Van Der Heide, B. (2012). "A social network as information: The effect of system generated reports of connectedness on credibility on Twitter". Computers in Human Behavior, 28(1), 199-206.

- Willis, A., Fisher, A., y Lvov, I. (2015). "Mapping networks of influence: tracking Twitter conversations through time and space". Participations: Journal of Audience \& Reception Studies, 12(1), 494-530.

- $\quad$ Yerva, S. R., Miklós, Z., y Aberer, K. (2012). "Quality-aware similarity assessment for entity matching in Web data". Information Systems, 37(4), 336-351.

- Zhao, W. X., Jiang, J., Weng, J., He, J., Lim, E. P., Yan, H., y Li, X. (2011). "Comparing twitter and traditional media using topic models". En: European Conference on Information Retrieval (pp. 338-349). Springer, Berlin, Heidelberg. 


\section{NOTAS:}

(1) Estos datos oficiales han sido recogidos en diversos medios de comunicación como El Mundo http://www.elmundo.es/tecnologia/2017/07/27/5979dc3146163fc6568b4674.html o El Economista https://www.eleconomista.com.mx/tecnologia/Usuarios-activos-de-Twitter-suben-a-328-millones-20170426-0157.html

(2) Acceso completo a los datos de la VIII Encuesta de Percepción Social de la Ciencia de FECYT disponible a través del siguiente enlace: https://www.fecyt.es/sites/default/files/news/ attachments/2017/07/epscyt2016 informe.pdf

(3) El panel estuvo compuesto por los siguientes expertos: Pilar Tigeras, Vicepresidenta adjunta de Cultura Científica del CSIC; José Manuel Sánchez-Ron, Catedrático de Historia de la Ciencia de la Universidad Autónoma de Madrid, Físico, divulgador y Académico de la RAE; Miguel Ángel Quintanilla Fisac, Catedrático de Lógica y Filosofía de la Ciencia de la USAL y exsecretario de Estado de Ciencia y Tecnología; Carlos Gacía Figuerola, profesor de la Universidad de Salamanca experto en análisis de redes; Felipe Romero, Socio Director y fundador de la consultora tecnológica The Cocktail Analysis, y Javier Pedreira, trabajador de la Casa de la Ciencias de A Coruña y co-fundador del blog de divulgación científica Microsiervos.

\section{CURRICULUM VITAE.}

\section{ANA VICTORIA PÉREZ-RODRÍGUEZ}

Doctora por la Universidad de Salamanca (España), donde imparte Comunicación de la Ciencia como profesora en el Master en Estudios Sociales de la Ciencia y la Tecnología. Actualmente es gerente en la Fundación 3CIN (Fundación Centro de Estudios para la Ciencia, la Cultura Científica y la Innovación), donde ejerce labores de investigación, comunicación y docencia. Sus áreas de investigación incluyen la comunicación de la ciencia, la participación pública en la ciencia, la política científica, la bibliometría y la ciencia abierta desde la perspectiva RRI (Responsible, Research and Innovation). Tiene más de 15 años de experiencia profesional como periodista de ciencia.

\section{CRISTINA GONZÁLEZ-PEDRAZ}

Master en Investigación (MRes) en Comunicación, investigadora en la Fundación 3CIN (Fundación Centro de Estudios para la Ciencia, la Cultura Científica y la Innovación) y estudiante de doctorado en la Universidad de Valladolid (España), donde también ha sido profesora asociada. Ha participado en diversos proyectos de investigación competitivos sobre comunicación de la ciencia y comunicación digital, los cuales han derivado en comunicaciones en congresos, artículos y capítulos de libro. También tiene 10 años de experiencia profesional como periodista de ciencia.

\section{JOSÉ LUIS ALONSO BERROCAL}

Profesor Titular del Departamento de Informática y Automática de la Universidad de Salamanca (España). Imparte docencia en el grado en Información y Documentación, en el Máster en Sistemas Inteligentes y en el Máster en Estudios Sociales de la Ciencia y la Tecnología. Es miembro del Instituto Universitario de Estudios de la Ciencia y de la Tecnología y forma parte del Grupo de investigación reconocido ELECTRA (Edición y lectura electrónica, transferencia y recuperación automatizada de la información). Su campo de investigación es la Recuperación de Información en el web y la Cibermetría, en el que ha publicado numerosos artículos y monografías. Ha participado en abundantes congresos especializados de carácter internacional. 
ANA VICTORIA PÉREZ-RODRÍGUEZ, CRISTINA GONZÁLEZ-PEDRAZ, JOSÉ LUIS ALONSO BERROCAL: Twitter como herramienta de comunicación científica en España. Principales agentes y redes de comunicación. 